ARTICLE

\title{
Sequential role of RAD51 paralog complexes in replication fork remodeling and restart
}

\author{
Matteo Berti ${ }^{14}$, Federico Teloni (10) 2,4, Sofija Mijic (10) ${ }^{1}$, Sebastian Ursich ${ }^{1}$, Jevgenij Fuchs (1) 1 , \\ Maria Dilia Palumbieri ${ }^{1}$, Jana Krietsch', Jonas A. Schmid ${ }^{1}$, Edwige B. Garcin (10 ${ }^{3}$, Stéphanie Gon (D) ${ }^{3}$, \\ Mauro Modesti (i] ${ }^{3}$, Matthias Altmeyer (i) ${ }^{2 凶} \&$ Massimo Lopes (iD ${ }^{1 凶}$
}

Homologous recombination (HR) factors were recently implicated in DNA replication fork remodeling and protection. While maintaining genome stability, HR-mediated fork remodeling promotes cancer chemoresistance, by as-yet elusive mechanisms. Five HR cofactors the RAD51 paralogs RAD51B, RAD51C, RAD51D, XRCC2 and XRCC3 - recently emerged as crucial tumor suppressors. Albeit extensively characterized in DNA repair, their role in replication has not been addressed systematically. Here, we identify all RAD51 paralogs while screening for modulators of RAD51 recombinase upon replication stress. Single-molecule analysis of fork progression and architecture in isogenic cellular systems shows that the BCDX2 subcomplex restrains fork progression upon stress, promoting fork reversal. Accordingly, BCDX2 primes unscheduled degradation of reversed forks in BRCA2-defective cells, boosting genomic instability. Conversely, the CX3 subcomplex is dispensable for fork reversal, but mediates efficient restart of reversed forks. We propose that RAD51 paralogs sequentially orchestrate clinically relevant transactions at replication forks, cooperatively promoting fork remodeling and restart.

\footnotetext{
${ }^{1}$ Institute of Molecular Cancer Research, University of Zurich, Winterthurerstrasse 190, 8057 Zurich, Switzerland. ${ }^{2}$ Department of Molecular Mechanisms of Disease, University of Zurich, Winterthurerstrasse 190, 8057 Zurich, Switzerland. ${ }^{3}$ Cancer Research Center of Marseille; CNRS; Inserm; Institut PaoliCalmettes; Aix-Marseille Université, 27 Bd Leï Roure, CS 30059, 13273 Marseille, France. ${ }^{4}$ These authors contributed equally: Matteo Berti, Federico Teloni.

凶email: matthias.altmeyer@dmmd.uzh.ch; lopes@imcr.uzh.ch
} 
rotecting the integrity of replicating chromosomes is crucial to maintain genome stability and to avoid cellular transformation. Mutations impairing the replication stress response predispose to cancer $^{1}$. However, the same mutations often impair the cellular responses to genotoxic treatments, offering important windows of opportunity for effective cancer chemotherapy by DNA replication interference ${ }^{2}$.

Replication fork reversal-i.e., the active conversion of replication forks into four-way junctions-has recently emerged as a global and genetically controlled response to various forms of endogenous and exogenous replication stress ${ }^{3-5}$. These transient replication intermediates can be efficiently restarted 6,7 and their function was proposed to slow down replication forks under unfavorable conditions, thereby limiting ssDNA accumulation and fork breakage ${ }^{8-10}$. Replication fork reversal requires specialized enzymes, such as the DNA translocases SMARCAL1, ZRANB3, and HLTF ${ }^{11-14}$, and the central recombinase RAD51 ${ }^{5}$. Genetic modifications affecting fork reversal typically result in unrestrained fork progression upon genotoxic stress, highlighting the relevance of fork remodeling for active fork slowing $5,12,15$. RAD51 function in regulating fork progression and remodeling extended the role of homologous recombination (HR) factors from classical double-stranded break (DSB) repair to the replication stress response ${ }^{16}$. Accordingly, other HR factors-like BRCA1 and BRCA2-protect stalled forks from excessive nucleolytic degradation by promoting efficient RAD51 loading $9,17,18$. This function in replication stress is genetically uncoupled from their canonical role in DSB repair ${ }^{14,17,19}$, is associated with the exquisite chemosensitivity of BRCA-deficient cells $^{20}$ and has been recently extended to additional HR factors ${ }^{21}$. Importantly, this deregulated degradation of nascent DNA is also genetically dependent on fork remodeling, as regressed arms are mandatory entry points for DNA degrading enzymes ${ }^{13,14,22,23}$. Moreover, the HR protein RAD52 was recently shown to modulate fork stability and restart, by limiting SMARCAL1dependent fork reversal or regulating reversed fork processing by MRE11 14,24. Collectively, these recent observations have implicated the RAD51 recombinase and other HR factors both in the formation and in the stability of reversed replication forks ${ }^{5,14}$. The latter function is BRCA2-dependent and reflects protection of the DNA end at the fourth regressed arm. However, the role of RAD51 in reversed fork formation is still enigmatic, as fork reversal does not require BRCA2, nor stable RAD51 nucleofilament formation ${ }^{14}$, thereby challenging canonical models of RAD51 function ${ }^{16}$. Molecular understanding of these clinically relevant transactions on replicating chromosomes requires further mechanistic investigation, particularly on the role of alternative $H R$ mediators and on their functional interaction with RAD51.

Besides RAD51 and its meiotic counterpart DMC1, five additional mammalian paralogs of bacterial RecA were discovered two decades ago, either by DNA sequence alignments (RAD51B, RAD51C, and RAD51D) or by functional complementation of radiation sensitive Chinese hamster ovary $(\mathrm{CHO})$ mutant cells (XRCC2 and XRCC3) ${ }^{25}$. These proteins display limited sequence homology to each other and to RAD51, and are generally reported as classical RAD51 paralogs. The Shu complex, which regulates $\mathrm{HR}$ in mitosis and meiosis, was recently shown to include a sixth RAD51 paralog, i.e., SWSAP125-27. Classical RAD51 paralogs were proposed to form two biochemically and functionally distinct subcomplexes, i.e. the RAD51B-RAD51CRAD51D-XRCC2 complex (BCDX2) and the RAD51C-XRCC3 complex (CX3), showing common, but also distinct biochemical properties ${ }^{28-32}$. Ablation of these proteins in mice resulted in embryonic lethality and functional studies on these factors were thus largely based on gene inactivation in p53-deficient CHO cells and chicken DT40 B-lymphocytes ${ }^{33-39}$. Overall, these studies uncovered important roles for these proteins in genome stability and DSB response, via modulation of RAD51. Recent biochemical data on the dimeric RAD51 paralog complexes in S. cerevisiae and C. elegans-Rad55/Rad57 and RFS-1/RIP-1, respectively-supported this concept, by showing that RAD51 paralogs protect RAD51 nucleofilaments from antirecombinase activities ${ }^{40}$ and remodel these filaments to promote strand exchange reactions ${ }^{41,42}$. However, whether similar biochemical properties can be associated with the different subcomplexes of human RAD51 paralogs is still unknown.

siRNA-mediated depletion of the human proteins suggested different roles for the two RAD51 paralog subcomplexes at early (BCDX2) versus late (CX3) stages of HR-mediated DSB repair ${ }^{43-45}$ Very recently, a panel of RAD51 paralog gene ablations was produced by CRISPR-Cas 9 in cancerous and non-transformed human cell lines ${ }^{46}$. While $R A D 51 B$ inactivation lead to comparatively mild phenotypes, genetic ablation of any of the other paralogs resulted in drastic impairment of HR-mediated DSB repair proficiency in all tested cell lines ${ }^{46}$.

Mutations in several RAD51 paralog genes have been associated with increased susceptibility to breast and ovarian cancer $^{47-51}$. Moreover, hypomorphic mutations in $R A D 51 C$ and $X R C C 2$ were linked to Fanconi anemia, a rare human disorder linked to defective replication-coupled repair of specific DNA lesions in the bone marrow ${ }^{52,53}$. These recent findings revived the interest in these accessory HR factors, promoting new mechanistic studies to unravel their precise role in DNA replication and genome stability. Interestingly, all human RAD51 paralogs were shown to associate with nascent $\mathrm{DNA}^{54}$, but the mechanistic role (s) of these factors in replication were not investigated systematically. $\mathrm{CHO}$ or DT40 cell lines carrying different mutations in individual genes-i.e., RAD51C, XRCC2, and XRCC3-displayed specific defects in replication fork progression and stability ${ }^{54-56}$. Furthermore, based on shRNA-mediated downregulation, human XRCC2 was recently proposed to modulate fork progression also in human cells, as a specific response to limited nucleotide availability ${ }^{57}$. Overall, our mechanistic understanding of the role of human RAD51 paralogs at replication forks is still incomplete and requires a systematic analysis of these factors in isogenic backgrounds.

Here, all classical human RAD51 paralogs are identified in a high-content microscopy screen for RAD51 modulation upon mild replication stress. Combining these cytological data with single-molecule investigations on replication intermediates in multiple cellular systems, we find that the BCDX2 complex assists RAD51 in reversed fork formation, limiting fork speed upon DNA damage and mediating stalled fork degradation in BRCA2defective cells. Conversely, the CX3 complex-albeit dispensable for fork reversal and fork protection-is found to mediate efficient restart of previously reversed forks.

\section{Results}

A screen for RAD51 foci in CPT-treated cells identifies RAD51 paralogs. RAD51 is readily detected on chromatin as nuclear foci in human osteosarcoma U2OS cells even during unperturbed replication. To identify key modulators of the RAD51 recombinase at challenged replication forks, we subjected U2OS cells to a short (45 min) treatment with low dose of the Topoisomerase I inhibitor camptothecin (CPT, $50 \mathrm{nM})$, which was previously shown to induce marked fork slowing and frequent fork rever$\mathrm{sal}^{58}$. As in these conditions induction of DSBs is undetectable ${ }^{58}$, RAD51 foci likely mark HR-mediated replication fork remodeling events occurring at stalled, yet-unbroken forks (Fig. 1a). We then designed a targeted small interfering RNA (siRNA) library, using 
a
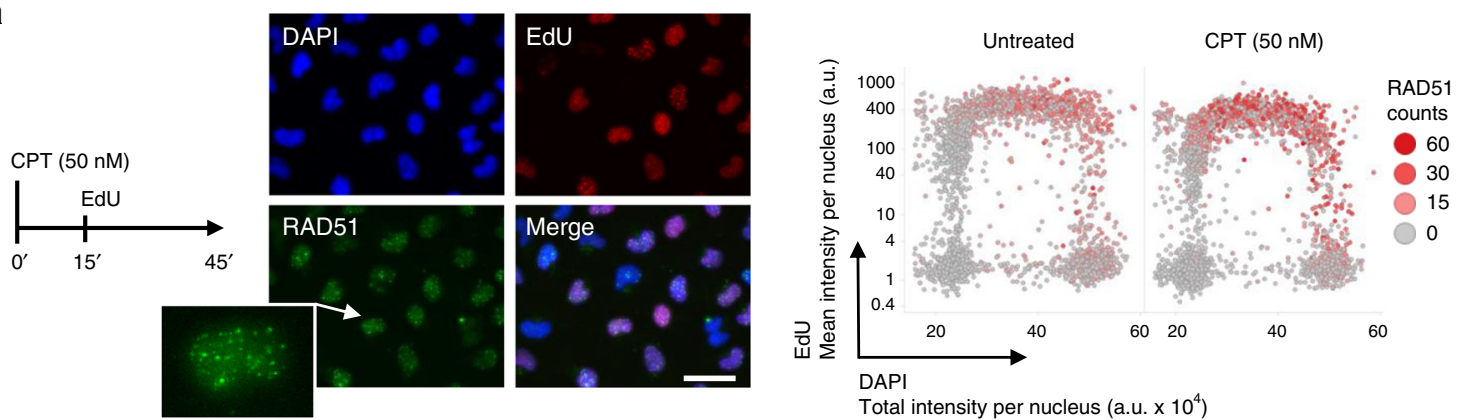

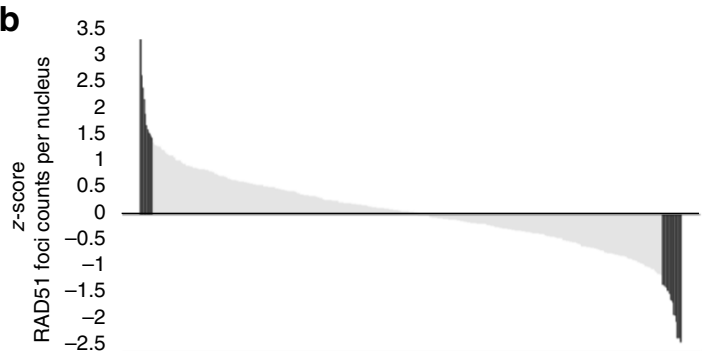

Gene name (ranked)

d

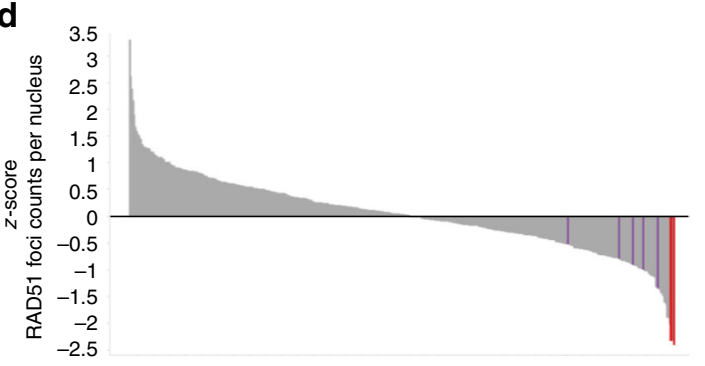

Gene name (ranked)

f

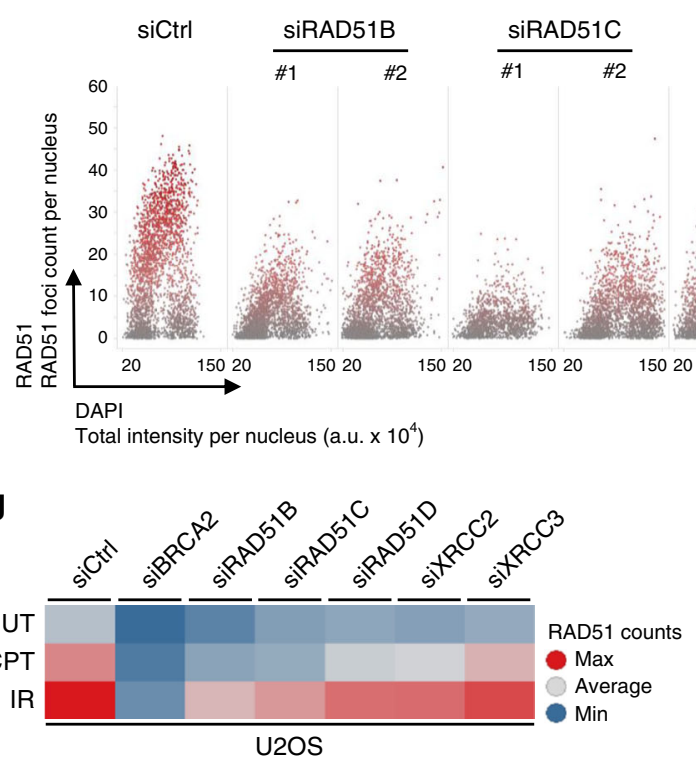

three individual siRNAs against each target gene and covering approximately 450 factors that were found to be enriched at replication forks by immunoprecipitation of newly synthesized DNA and mass spectrometric analysis of the associated proteins ${ }^{59-61}$. CPT-treated cells were labeled with EdU and analysed by high-content microscopy for quantitative image-based cytometry $(\mathrm{QIBC})^{62,63}$, which allows simultaneous assessment of cell-

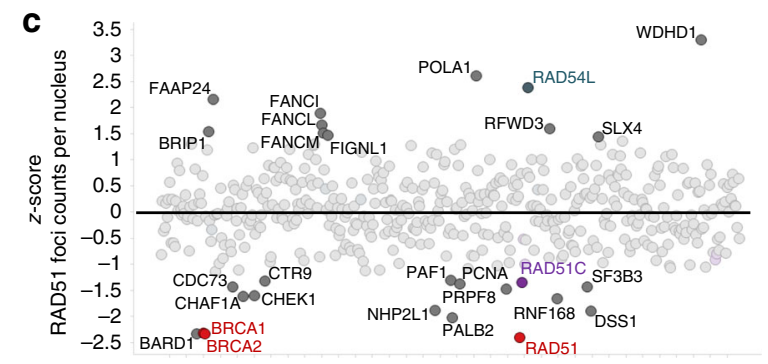

Gene name (alphabetically)

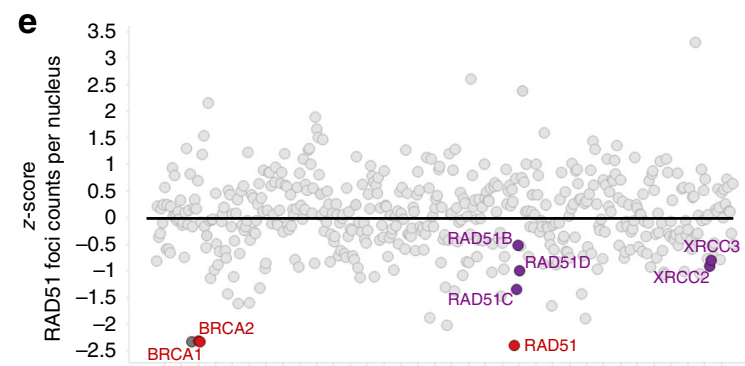

Gene name (alphabetically)
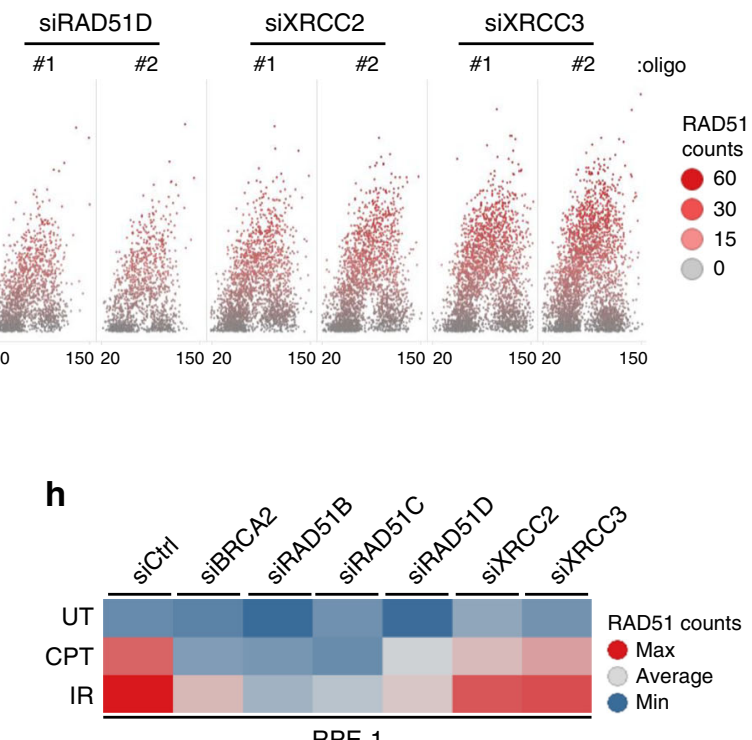

cycle distribution, replication competence, as well as number and intensity of RAD51 foci. We selected an early time point after siRNA transfection $(48 \mathrm{~h})$, in order to limit cell-cycle effects due to prolonged depletion of essential factors. In these hypomorphic conditions, only depletion of key replication factors (e.g., PCNA) led to replication impairment, while most other siRNAs did not impact replication competence and cell proliferation, including 
Fig. 1 QIBC-based screen identifies RAD51 paralogs as important modulators of RAD51 foci formation upon mild replication stress. a QIBC-based screen layout. Left: screen experimental condition. U2OS cell were treated with $50 \mathrm{nM} \mathrm{CPT}$ for 45 min and replicating cells were concomitantly labeled with EdU for $30 \mathrm{~min}$. Center: representative immunofluorescence image (scale-bar, $50 \mu \mathrm{m}$ ). Right: representative scatter plot showing cell-cycle distribution (based on DNA content and EdU incorporation) of RAD51 foci in untreated and CPT-treated U2OS cells. The experiment shown was performed twice yielding similar results. $\mathbf{b}$ Screen gene ranking based on the RAD51 foci z-score. c z-score plot of positive $(z$-score $<-1.5)$ and negative $(z$-score $>1.5)$ RAD51 modulators (marked in gray). Color code as in d, e. d, e z-score for the five classical RAD51 paralogs (marked in purple) and positive controls (marked in red). $\mathbf{f}$ Validation of the RAD51 paralogs influence on RAD51 foci formation upon $50 \mathrm{nM} \mathrm{CPT.} \mathbf{g}$, $\mathbf{h}$ Differential effect of RAD51 paralogs on RAD51 foci formation in untreated (UT), CPT- ( $50 \mathrm{nM}$ for $45 \mathrm{~min}$ ) and IR- ( $5 \mathrm{~Gy}, 2 \mathrm{~h}$ recovery) treated U2OS and RPE-1 cells. Source data for a-f are provided in the Source Data file.

those targeting essential HR factors like BRCA1 (Supplementary Fig. 1a). Reassuringly, when ranked according to their deviation from the mean (i.e., $\mathrm{z}$-score), known positive (BRCA1, BRCA2, BARD1, PALB2) and negative regulators (RAD54L) of RAD51 scored among the top hits at either end of the distribution (Fig. 1b, c, Supplementary Data 1 and 2), with all three siRNAs showing consistent results (Supplementary Data 2). However, presumably because of the hypomorphic conditions chosen for the screen, siRNAs targeting genes of other known antirecombinase activities (i.e., RADX/CXorf57, FBH1/FBXO18, RECQ5, and PARI/C12orf48) did not show strong effects on RAD51 accumulation (Supplementary Fig. 1b-c; Supplementary Data 1 and 2). Furthermore, although RAD51 has been previously involved in different steps of fork remodeling and protection, siRNAs targeting genes for key fork remodeling factors (i.e., SMARCAL1, ZRANB3, HLTF, and RECQ1/RECQL) did not significantly affect RAD51 foci number and intensities in our screening conditions (Supplementary Fig. 1b-c). Besides the possibility of incomplete downregulation, this result may also reflect intrinsic ambiguities associated with RAD51 foci measurements as sole readout. Impairing fork remodeling activities may not only negatively affect RAD51 binding to forks, but can also induce fork breakage even at these minimal CPT doses 5,58 , thereby enhancing RAD51 accumulation in foci.

Despite these potential caveats and its inherently noncomprehensive nature, our screen identified several new or uncharacterized modulators of RAD51 chromatin binding upon replication stress, showing strong positive $(>1.5)$ or negative $(<1.5)$ z-scores (Fig. 1c, Supplementary Data 1). Here, we focused our attention on the five classical RAD51 paralogs, which all displayed a negative $\mathrm{z}$-score in the experimental conditions of our screen (Fig. 1d, e). SWSAP1-encoding another noncanonical RAD51 paralog $25-27$ - was not among the targeted genes in our siRNA library, but downregulation of its interaction partner SWS1 led to defects in RAD51 foci comparable to those observed for the other RAD51 paralogs (Supplementary Fig. 1b-c, Supplementary Data 1), in agreement with the recently reported involvement of the Shu complex in the replication stress response ${ }^{64}$.

In light of the established clinical relevance of the classical RAD51 paralogs and their elusive role in the replication stress response, we decided to focus on and explore this set of genes. We validated the screen results in U2OS cells using two individual siRNAs, testing also-besides low CPT treatments-untreated conditions and ionizing radiation. In these analyses, short-term (48 h) depletion of all classical RAD51 paralogs consistently led to reduced RAD51 foci counts in replicating cells, while not significantly affecting cell cycling and replication competence (Fig. 1f, g and Supplementary Fig. 1d). In agreement with previous reports ${ }^{45}$, we noticed that XRCC3 downregulation showed milder effects on RAD51 foci, compared to inactivation of the other RAD51 paralogs. This difference was also observed when performing validation experiments in untransformed human retinal epithelial cells (RPE-1, Fig. 1h) and is particularly evident in S phase cells upon mild CPT treatment (Supplementary Fig. 1e). As XRCC3 and RAD51C form a specific protein complex (CX3), while all other factors assemble in a second multimeric RAD51 paralog complex (BCDX2), these results pointed to separate functional roles of the two complexes in the replication stress response, as suggested for DSB repair ${ }^{45}$.

BCDX2 promotes fork slowing and remodeling upon mild genotoxic stress. To investigate the functional role of BCDX2 and CX3 in the replication stress response, we first monitored the stability of the two complexes upon single-component downregulation in U2OS cells by two independent siRNAs (Fig. 2a, b). In keeping with previous reports ${ }^{45}$, RAD51C downregulation affected protein levels of all components of both complexes, thus potentially abolishing both BCDX2 and CX3 activities. In contrast, $R A D 51 D$ downregulation did leave CX3 levels unaffected, thus allowing the assessment of the specific functional role of BCDX2. Conversely, XRCC3 downregulation had only minor effects on RAD51C levels and should thus specifically affect CX3 function (Fig. 2a, b). We therefore focused our functional analysis on these three protein depletions, to explore specific roles of the two complexes upon mild replication interference (Fig. 2a). We first used an established nascent DNA labeling protocol to monitor by DNA fiber spreading active replication fork slowing upon mild treatment with $\mathrm{CPT}^{58}$. RAD51C and RAD51D downregulation by two independent siRNA sequences drastically impaired active fork slowing, leading to unrestrained fork progression in the presence of CPT, both as measured by fiber track length and as ratios between the differentially labeled nascent DNA (Fig. 2c, and Supplementary Fig. 2a-c). Conversely, XRCC3 downregulation did not significantly affect CPT-induced fork slowing (Fig. 2c, and Supplementary Fig. 2a-c). We then performed similar experiments in U2OS cells where the same three genes had been knocked-out (KO) by CRISPR-Cas9 ${ }^{46}$. Compared to RAD51 paralog downregulation by siRNA (Supplementary Fig. 1a), all KO cells-including XRCC3-KO-displayed a more drastic reduction of endogenous and drug-induced RAD51 foci (Supplementary Fig. 2d), likely reflecting full and prolonged inactivation of these factors. Nonetheless, all tested KO cells led to very similar observations when compared to downregulated cells in terms of protein level interdependency and replication fork progression phenotypes, with $R A D 51 C-K O$ and $R A D 51 D-K O$ cells, but not XRCC3-KO cells, displaying unrestrained fork progression in CPT (Fig. 2d, e). Importantly, unrestrained fork progression in $R A D 51 C-K O$ and $R A D 51 D-K O$ cells was readily complemented by stable expression of a FLAG-tagged version of the missing protein (Fig. 2d, e). Finally, we confirmed very similar effects on CPT-induced fork slowing by siRNA-mediated downregulation of $R A D 51 C, R A D 51 D$, and XRCC3 in the nontransformed RPE-1 human cell line (Supplementary Fig. 2e). Overall, these data strongly suggest that BCDX2, but not $\mathrm{CX} 3$, mediates active fork slowing upon mild genotoxic stress.

Active fork slowing is linked to replication fork reversal (Fig. 2f) and depends on the RAD51 recombinase and multiple 
a

BCDX2 complex

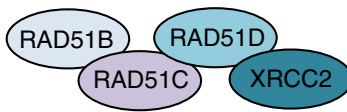

CX3 complex
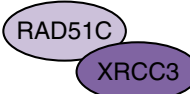

\begin{tabular}{c|c|c|}
$\begin{array}{c}\text { Downregulation/ } \\
\text { inactivation }\end{array}$ & \multicolumn{2}{|c|}{ Stability/function } \\
BCDX2 & CX3 \\
\hline RAD51C & $\boldsymbol{x}$ & $\boldsymbol{x}$ \\
RAD51D & $\boldsymbol{x}$ & $\checkmark$ \\
XRCC3 & $\checkmark$ & $\mathbf{x}$ \\
\hline
\end{tabular}

b

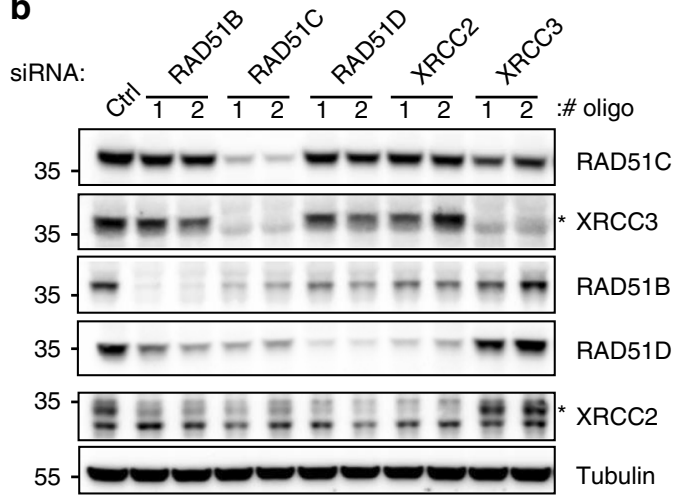

C

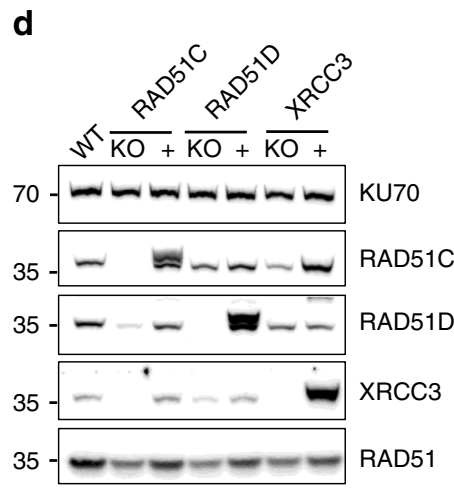

e
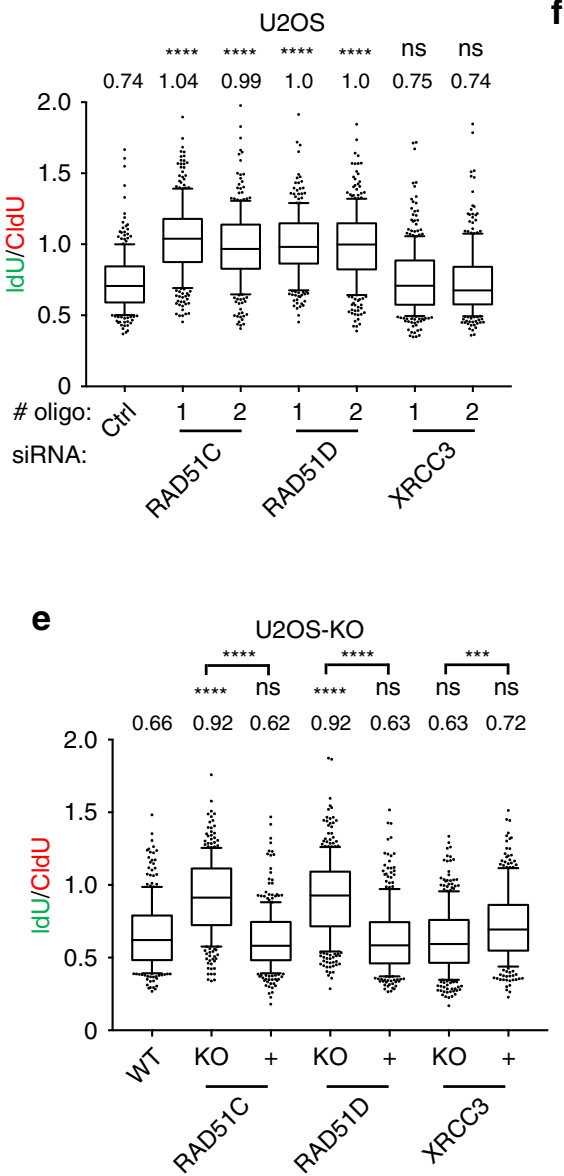

f

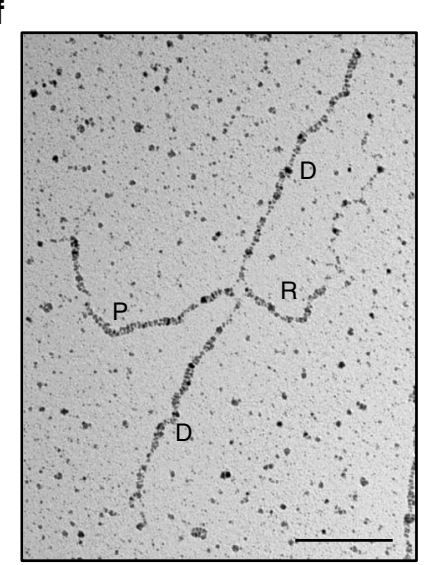

g

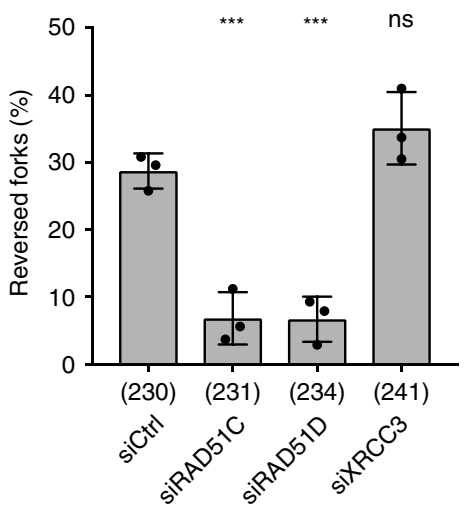

Fig. 2 BCDX2 paralog complex, but not CX3, promotes replication fork slowing and reversal upon mild CPT treatment. a Rationale for focusing on RAD51C, RAD51D, and XRCC3 downregulation, to perform functional studies on BCDX2 and CX3 complexes during replication stress. Top: schematic of the two main RAD51 paralog complexes. Bottom: differential effects of RAD51C, RAD51D, and XRCC3 inactivation on paralog complex stability/function. b Western blot analysis of RAD51 paralogs upon $48 \mathrm{~h}$ downregulation with two different siRNAs. Ctrl, control siRNA; Tubulin, loading control. asterisk, specific band. In $\mathbf{b}$ and $\mathbf{d}$ multiple gels/blots were processed in parallel, ensuring equal and comparable loading across gels. The experiment was performed twice yielding similar results. c DNA fiber analysis of U2OS cells $48 \mathrm{~h}$ after transfection with a control siRNA (siCtrl) or with siRNAs targeting RAD51C, RAD51D, and XRCC3. Top-left: schematic of the CldU/IdU pulse-labeling protocol used to evaluate fork progression upon $50 \mathrm{nM} C P T$ treatment. Bottomleft: representative DNA fibers from each genetic condition. Left: IdU/CIdU ratio is plotted as a readout of fork progression. In $\mathbf{c}$ and $\mathbf{e}$, the numbers indicate the mean value; a minimum of 150 forks was scored in two independent experiments yielding similar results. Bounds of box are 25-75th percentile, center shows the median, whiskers indicate the 10-90 percentiles, data points outside this range are drawn as individual dots. Statistical analysis: Kruskal-Wallis test; ns not significant; ${ }^{* \star \star \star} p$-value $<0.0001,{ }^{* \star *} p$-value $=0.0003$. d Western blot analysis of RAD51 paralogs in Knock-Out (KO) U2OS cells and in cells reconstituted of the respective protein (+). KU70, loading control. The experiment was performed twice yielding similar results. e DNA fiber analysis of KO and reconstituted $(+)$ U2OS cells labeled as in $\mathbf{c}$. $\mathbf{f}, \mathbf{g}$ Frequency of reversed replication forks in U2OS cells transfected with control siRNA (Ctrl) or with siRNAs targeting RAD51C, RAD51D, or XRCC3 for $48 \mathrm{~h}$ and treated with $50 \mathrm{nM} \mathrm{CPT}(1 \mathrm{~h})$. $\mathbf{f}$ Electron micrograph of a representative reversed replication fork from CPT-treated U2OS cells (P parental duplex, D daughter duplexes, R regressed arm; scale-bar, $100 \mathrm{~nm}$ ). $\mathbf{g}$ Graph-bar showing mean and SD from three independent EM experiments. In brackets, total number of molecules analyzed per condition. Statistical analysis: one-way ANOVA followed by Bonferroni test; ns not significant; ${ }^{\star \star \star} p$-value $=0.0004$. Source data for $\mathbf{b}$-g are provided in the Source Data file. 
a

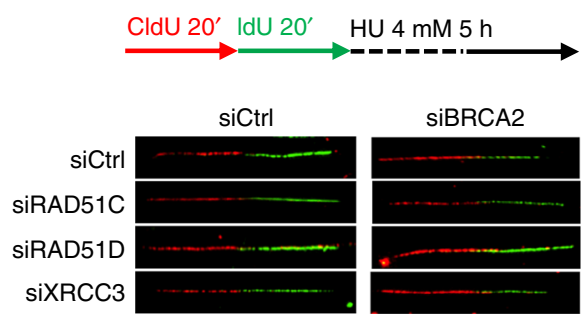

C

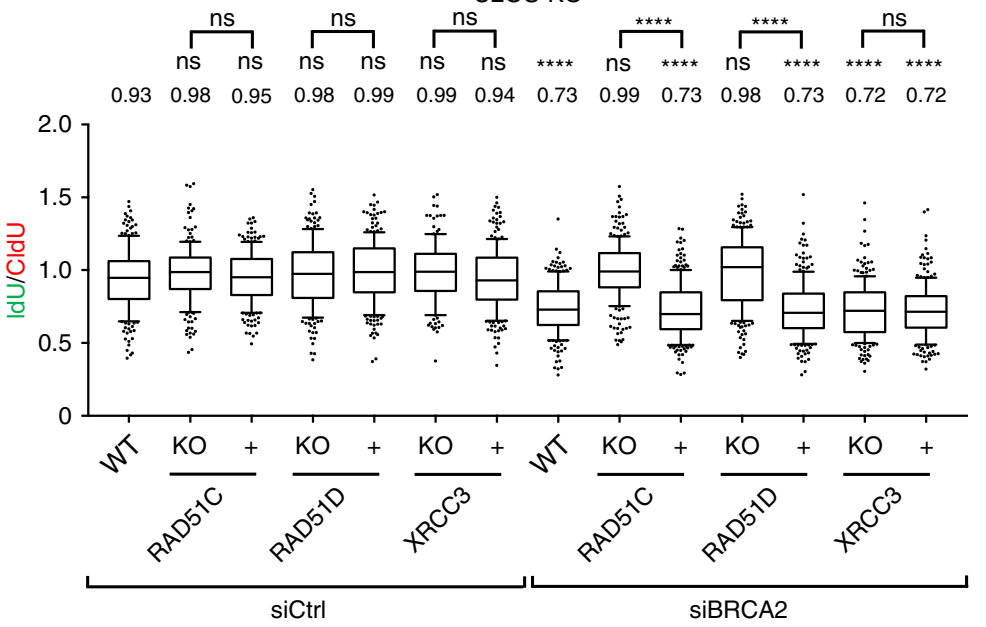

b

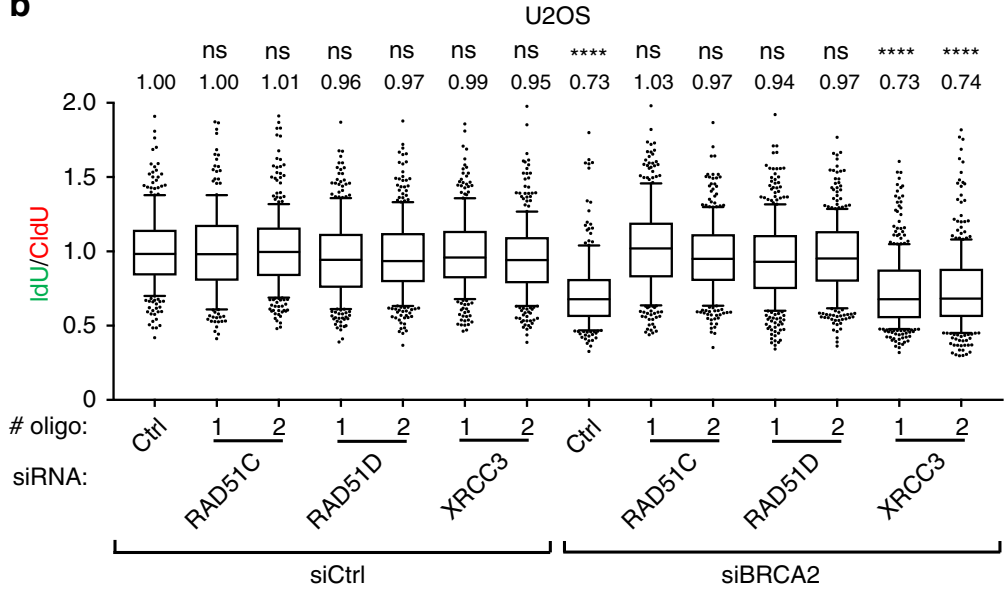

d

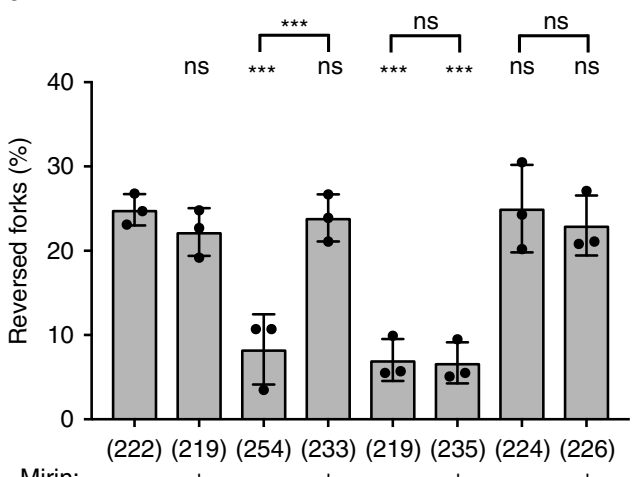

Mirin:

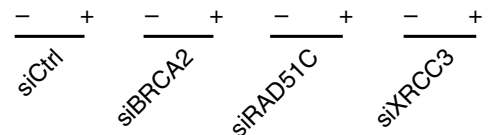

Fig. 3 BCDX2 complex, but not CX3, promotes reversed fork degradation in BRCA2-depleted U2OS cells. a, b DNA fiber analysis of U2OS cells double transfected with a control siRNA (Ctrl) or with siRNAs targeting RAD51C, RAD51D, and XRCC3 for $60 \mathrm{~h}$ and with BRCA2 siRNA for $48 \mathrm{~h}$. a Top: schematic $\mathrm{CldU} / \mathrm{IdU}$ pulse-labeling protocol to evaluate fork degradation upon $\mathrm{HU}$ treatment ( $4 \mathrm{mM}, 5 \mathrm{~h}$ ). Bottom: representative DNA fibers from each genetic condition. $\mathbf{b} \mathrm{IdU} / \mathrm{CldU}$ ratio is plotted as a readout of fork degradation. In $\mathbf{b}$ and $\mathbf{c}$, the numbers indicate the mean value; a minimum of 150 forks was scored in two independent experiments yielding similar results. Bounds of box are 25-75th percentile, center shows the median, whiskers indicate the $10-90$ percentiles, data outside this range are drawn as individual dots. Statistical analysis: Kruskal-Wallis test; ns not significant; ${ }^{\star \star \star \star} p$-value $<0.0001$. c DNA fiber analysis of $\mathrm{KO}$ and reconstituted (+) U2OS cells transfected with a control siRNA (Ctrl) or with BRCA2 siRNA for $48 \mathrm{~h}$ and consecutively labeled and $\mathrm{HU}$-treated as in a. IdU/CldU ratio is plotted as a readout of fork degradation. d Frequency of reversed replication forks in U2OS cells transfected with control siRNA (Ctrl) or with siRNAs targeting BRCA2, RAD51C, or XRCC3 for $48 \mathrm{~h}$ and treated for $5 \mathrm{~h}$ with $\mathrm{HU} 4 \mathrm{mM}$; where indicated $50 \mu \mathrm{M} \mathrm{Mirin}$ was added $1 \mathrm{~h}$ before $\mathrm{HU}$ treatment ( $6 \mathrm{~h}$ total treatment). Graph-bar depicts mean and SD from three independent EM experiments. In brackets, total number of molecules analyzed per condition. Statistical analysis: one-way ANOVA followed by Bonferroni test; ns not significant; ${ }^{\star \star \star} p$-value $=0.0003$. Source data for a-d are provided in the Source Data file.

DNA translocases $5,12,65$. Using an established EM method to visualize replication intermediates ${ }^{66}$, we indeed confirmed that downregulation of $R A D 51 C$ and $R A D 51 D$, but not of XRCC3, markedly impairs CPT-induced replication fork reversal (Fig. 2g, Supplementary Table 1a).

BCDX2 primes stalled fork degradation in BRCA2-defective cells. We then used the same validated cellular systems to further investigate the role of BCDX2 and CX3 in fork stability, using an established DNA fiber protocol where double-labeled ongoing forks experience prolonged stalling by $\mathrm{HU}$-induced nucleotide depletion (Fig. 3a) ${ }^{14,17}$. As reported, BRCA2-depleted cells displayed nascent DNA degradation at stalled forks, monitored as reduced ratio between second (IdU) and first (CldU) track length (Fig. 3a, b). Despite effective depletion of protein levels (Supplementary Fig. 3a)-which were sufficient to induce unrestrained fork progression in CPT (Fig. 2c)-downregulation of none of the tested RAD51 paralogs led to detectable fork degradation (Fig. 3a, b). However, downregulation of $R A D 51 C$ or $R A D 51 D$-but not of XRCC3-with two different siRNA sequences fully restored nascent DNA stability in BRCA2depleted cells (Fig. 3a, b and Supplementary Fig. 3a). Very similar results were obtained in RAD51C-KO and in RAD51D-KO U2OS cells, and re-expression of the missing protein restored the fork degradation phenotype upon BRCA2 depletion (Fig. 3c). Also in this cellular system, genetic inactivation of XRCC3 had no detectable effects on nascent DNA stability, in either BRCA2proficient or -deficient cells (Fig. 3c). Consistently, downregulation of $R A D 51 C$ or $R A D 51 D$-but not XRCC3-fully restored stalled fork stability also in BRCA2-depleted RPE-1 cells (Supplementary Fig. 3b-c). On one hand, these observations suggest that inactivation of any of the RAD51 paralogs does not 
affect stalled fork integrity in human cells. On the other hand, in light of the data in Fig. 2, they also suggest that BCDX2-but not CX3-mediates the formation of reversed forks, which serve then as entry points for nucleolytic degradation of nascent DNA in BRCA2-defective cells. EM visualization confirmed this hypothesis: as reported in different cellular systems ${ }^{13,14,22}$, BRCA2depleted U2OS cells displayed a marked reduction in HUinduced reversed fork frequency, which was readily suppressed by MRE11 inhibition using mirin (Fig. 3d, Supplementary Table 1b). Conversely, mirin did not restore reversed fork frequency upon RAD51C depletion and XRCC3 depletion had no significant effects (Fig. 3d, Supplementary Table 1b). These EM data on RAD51C closely resemble previous observations obtained by RAD51 depletion ${ }^{14}$ and, combined with the data in Fig. 3b-c, strongly suggest that $\mathrm{BCDX} 2$-but not $\mathrm{CX} 3$-is required for fork reversal, upstream of BRCA2-mediated stabilization of reversed forks.

\section{CX3 mediates efficient restart of reversed forks after stalling.} We next tested by DNA fiber spreading whether RAD51 paralogs assist the restart of stalled replication forks, measuring number and length of replicated tracks after HU removal (Fig. 4a). In our experimental conditions ( $24 \mathrm{~h}$ of siRNA-mediated downregulation), $R A D 51$ inactivation did not affect the efficiency of fork restart and slightly increased the velocity of restarting forks (Fig. 4a and Supplementary Fig. 4a). This likely reflects impaired fork remodeling associated with RAD51 depletion, as non-reversed forks may be faster in restarting DNA synthesis once nucleotide levels are restored, possibly by efficient replication fork repriming events. Accordingly, downregulation of RAD51C or RAD51D-which are also required for fork reversal (Figs. 2 and 3)-led to similar mild effects on fork restart (Fig. 4a and Supplementary Fig. 4a). Conversely, XRCC3 depletion drastically increased the fraction of forks failing to restart DNA synthesis and delayed progression of the restarting forks (Fig. 4a and Supplementary Fig. 4a). Very similar effects on efficiency and velocity of fork restart were obtained in U2OS cells carrying genetic ablation of these genes and all effects were fully complemented by re-expression of the missing protein (Supplementary Fig. $4 \mathrm{~b}-\mathrm{c}$ ). As XRCC3 is not required for fork reversal (Figs. 2 and 3), we reasoned that XRCC3-depleted cells might be specifically impaired in their ability to restart forks that had previously been reversed. Indeed, co-depletion of RAD51 in XRCC3-depleted U2OS cells fully rescued efficiency and velocity of fork restart (Fig. $4 \mathrm{~b}$ and Supplementary Fig. 4d). Similarly, XRCC3 depletion impaired fork restart in ZRANB3-proficient, but not in ZRANB3-deficient cells (Fig. 4c and Supplementary Fig. 4e). Moreover, also RAD51D depletion rescued fork restart efficiency and velocity in XRCC3depleted cells (Fig. 4d, Supplementary Fig. 4f), further supporting the role of BCDX2 in fork reversal, upstream of XRCC3-mediated restart. Finally, as XRCC3 is destabilized in RAD51C-depleted cells (Fig. 2b), RAD51C downregulation itself represents a third condition that impairs fork reversal and rescues fork restart in the absence of XRCC3 (Fig. 4a). Overall, these data establish a specific role for the CX3 complex in replication fork restart, downstream of RAD51-, BCDX2-, and ZRANB3-dependent fork reversal.

BCDX2 fuels chromosomal instability in BRCA2-defective cells. As reversed forks are the entry points for unscheduled fork degradation in BRCA2-depleted cells, we finally tested whether genetic impairment of RAD51C and RAD51D-which are required for fork reversal-may restore genome stability in BRCA2-defective cells. In metaphase spreads, depletion of RAD51 paralogs per se did not detectably increase chromosomal abnormalities (Fig. 5a). However, depletion of RAD51C or
RAD51D-but not of XRCC3-suppressed the chromosomal instability associated with BRCA2 depletion (Fig. 5a), suggesting that efficient fork remodeling by BCDX2 engages BRCA2 in a crucial genome maintenance function.

\section{Discussion}

The recent discoveries that mutations in classical RAD51 paralog genes predispose to cancer and Fanconi anemia ${ }^{47-53}$ encouraged new mechanistic studies on these HR accessory factors, particularly focused on the human proteins and their possible role in the replication stress response. Human RAD51 paralogs were first expressed and purified over two decades ago, leading to the identification of two main complexes: BCDX2 (composed of RAD51B, RAD51C, RAD51D, XRCC2) and CX3 (composed of RAD51C and XRCC3). However, biochemical insights have remained relatively scarce to date. Furthermore, until very recently, genetic investigations on these proteins were also largely limited to single siRNAs or mutated cell lines in specific model systems, calling for more systematic genetic investigations of these proteins in isogenic human systems, particularly with respect to their role upon replication stress.

Here, we selectively inactivated the BCDX2 complex (via $R A D 51 D$ inactivation), the CX3 complex (via XRCC3 inactivation), or both complexes (via RAD51C inactivation) in U2OS and RPE-1 human cells, and performed single-molecule investigations on replication intermediates. We show that the BCDX2 complex drives reversed replication fork formation, presumably by assisting the central recombinase RAD51, which was previously shown to mediate this step of fork remodeling 5,14 . Differently from replication fork protection and HR-mediated DSB repair, reversed fork formation does not require stable RAD51 nucleofilaments ${ }^{14}$. This suggests that BCDX2 may assist DNA translocases and RAD51 in driving parental strand reannealing, while partially replacing RPA on ssDNA stretches accumulating at stalled forks (Fig. 5b). RAD51 paralogs were shown in yeast and C. elegans to biochemically remodel preassembled RAD51 nucleofilaments ${ }^{40-42}$. It is possible that RAD51 paralogs enhance and stabilise spontaneous RAD51 monomer binding to short ssDNA stretches at stalled forks, thus supporting the formation of a metastable nucleofilament, with sufficient flexibility to sustain the high torsional constrains at replication forks and promote fork reversal. Alternatively, RAD51 paralogs might stimulate the spontaneous formation of BRCA2independent short stretches of RAD51 monomers at stalled forks by preventing the action of human antirecombinase activities ${ }^{40}$. However, our data do not exclude other possible RAD51independent mechanisms by which BCDX2 may foster the reversal reaction, such as intrinsic strand annealing activities ${ }^{29}$ or specific interactions with different enzymatic activities (e.g., DNA translocases) and cofactors involved in fork reversal. While most reported biochemical assays of fork reversal were performed in the absence of ssDNA binding proteins, the addition of RPA or RAD51-when tested-had profound effects on the efficiency and/or directionality of these reactions ${ }^{67,68}$. Holistic biochemical reconstitution of these important transactions at the replication fork seems necessary to elucidate the precise molecular mechanism by which BCDX2 and RAD51 collaborate with DNA translocases to drive efficient fork reversal.

As already shown for RAD51, which plays key genetically uncoupled roles both in the formation and in the protection of reversed forks ${ }^{14,16,17}$, our data do not exclude that BCDX2 plays also a crucial role in protection and restart of stalled replication forks (Fig. 5b). However, as for RAD51, this role may be masked by its crucial upstream function in replication fork reversal. As exemplified for RAD51 by the T131P separation-of-function mutant ${ }^{14}$, it is likely that hypomorphic 
a
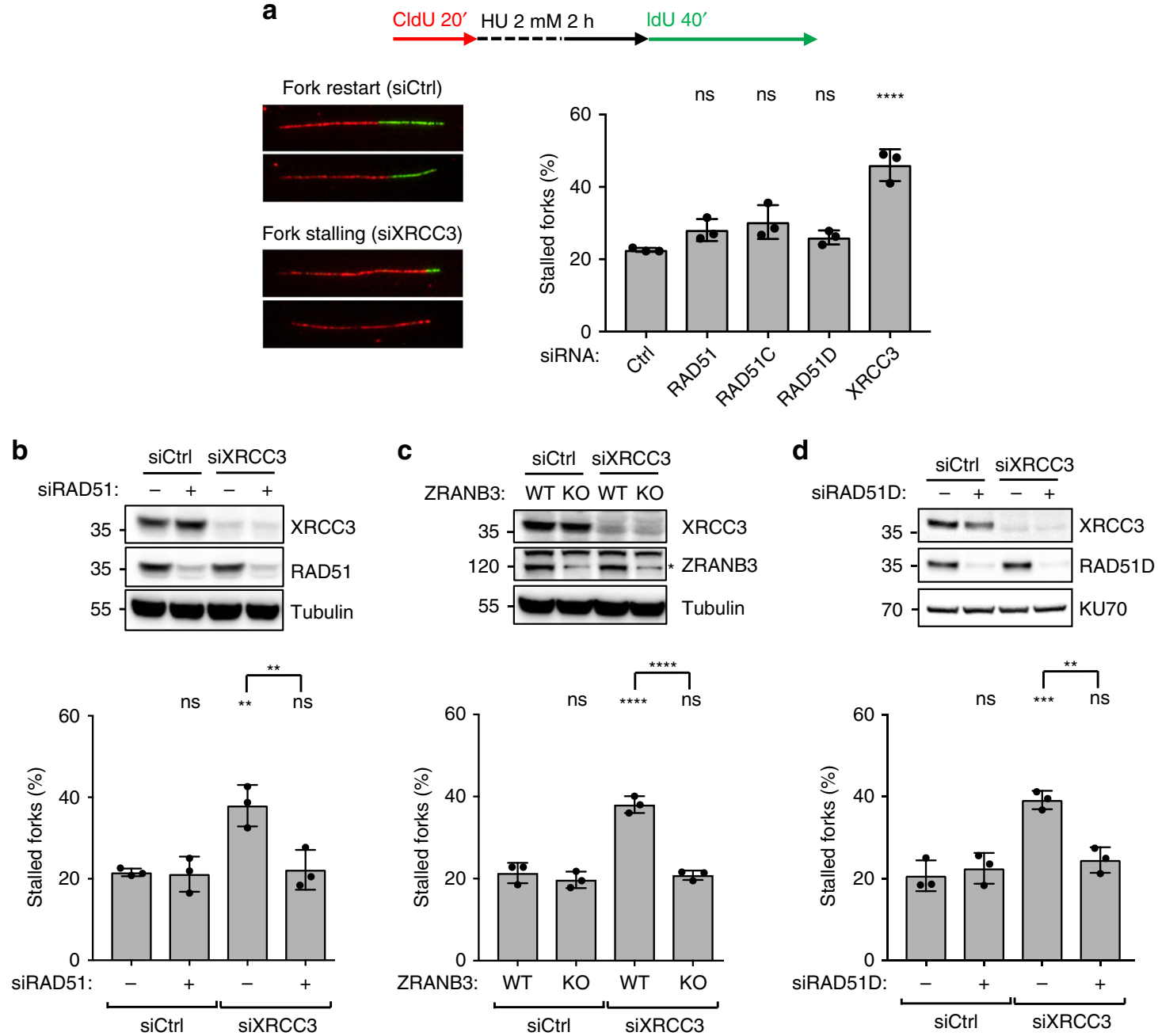

Fig. 4 CX3 complex promotes reversed fork restart in U2OS cells. a DNA fiber analysis of U2OS cells transfected for $24-48 \mathrm{~h}(24 \mathrm{~h}$ for $R A D 51,48 \mathrm{~h}$ for all the other genes) with a control siRNA (Ctrl) or with siRNAs targeting RAD51, RAD51C, RAD51D, and XRCC3 to investigate replication fork restart upon HU. Top: schematic CldU/IdU pulse-labeling protocol to evaluate fork restart upon HU treatment $(2 \mathrm{mM}, 2 \mathrm{~h})$. Bottom-left: representative stalled and restarting forks. Bottom-right: frequency of fork stalling for each genetic condition. In a-d, the graph-bar depicts mean and SD from three independent experiments. Statistical analysis: one-way ANOVA followed by Bonferroni test; ns not significant; ${ }^{\star \star \star \star} p$-value $<0.0001$. b DNA fiber analysis of U2OS cells double transfected with a control siRNA (Ctrl) or with a siRNA targeting XRCC3 (48 h) or RAD51 (24 h) to investigate replication fork restart upon HU treatments; labeling protocol as in $\mathbf{a}$. Top: levels of indicated proteins, assessed by western blot; Tubulin, loading control. In $\mathbf{b}$ and $\mathbf{d}$ multiple gels/blots were processed in parallel, ensuring equal and comparable loading across gels. Bottom: frequency of fork stalling. ns not significant; ${ }^{\star \star} p$-value $=0.0068(1$ versus 3$)$ and 0.0087 (3 versus 4). c DNA fiber analysis of wild-type (WT) or ZRANB3 Knock-Out (KO) U2OS cells transfected with a control siRNA (Ctrl) or with siRNAs targeting XRCC3 $(48 \mathrm{~h})$ to investigate replication fork restart upon $\mathrm{HU}$ treatments; labeling protocol as in $\mathbf{a}$. Top: indicated protein levels, assessed by western blot; Tubulin, loading control; asterisk, specific band. Bottom: frequency of fork stalling. ns not significant; ${ }^{\star \star \star \star} p$-value $<0.001$. $\mathbf{d}$ DNA fiber analysis of U2OS cells double transfected with a control siRNA (Ctrl), with siRNAs targeting XRCC3 (48 h) or RAD51D (60 h) to investigate replication fork restart upon HU treatments; labeling protocol as in a. Top: indicated protein levels, assessed by western blot; KU70, loading control. Bottom: frequency of fork stalling. ns not significant; ${ }^{\star \star} p$-value $=0.0024 ;{ }^{\star \star \star} p$-value $=0.0007$. Source data for a-d are provided in the Source Data file.

conditions of RAD51 paralog inactivation and/or different levels and regulations of these proteins in different model systems may explain different phenotypes in fork progression and degradation upon inactivation of BCDX2 and CX3 components ${ }^{54-57}$. In this context, it will be paramount to use isogenic replacement systems to test fork progression, remodeling and stability with specific RAD51 paralog mutants linked with cancer and Fanconi anemia ${ }^{47-53}$, to reveal the specific function(s) of these proteins in preventing human disease.

Our data uncover a role for the CX3 complex in the restart of reversed replication forks, establishing a sequential engagement of different RAD51 paralog complexes in stalled fork remodeling and reactivation (Fig. 5b). Interestingly, a downstream role for
CX3 - with respect to BCDX2-had been hypothesized also in classical HR-mediated repair of DSBs, based on the limited requirement of XRCC3 for RAD51 foci formation ${ }^{45}$ and on the Holliday junction resolution activity found associated with the CX3 complex ${ }^{69}$. Recent data with CRISPR-KO cells challenge this model $^{46}$, but may also reflect long-term effects of chronic XRCC3 inactivation and cell adaptation mechanisms to avoid the resultant accumulation of toxic recombination intermediates. While we did not succeed in monitoring directly the recruitment of RAD51 paralogs to replication forks with currently available tools, XRCC3 was previously shown to bind nascent DNA with a delayed kinetic compared to BCDX2 subunits ${ }^{54}$, which is consistent with the sequential model proposed here (Fig. 5b). 
a

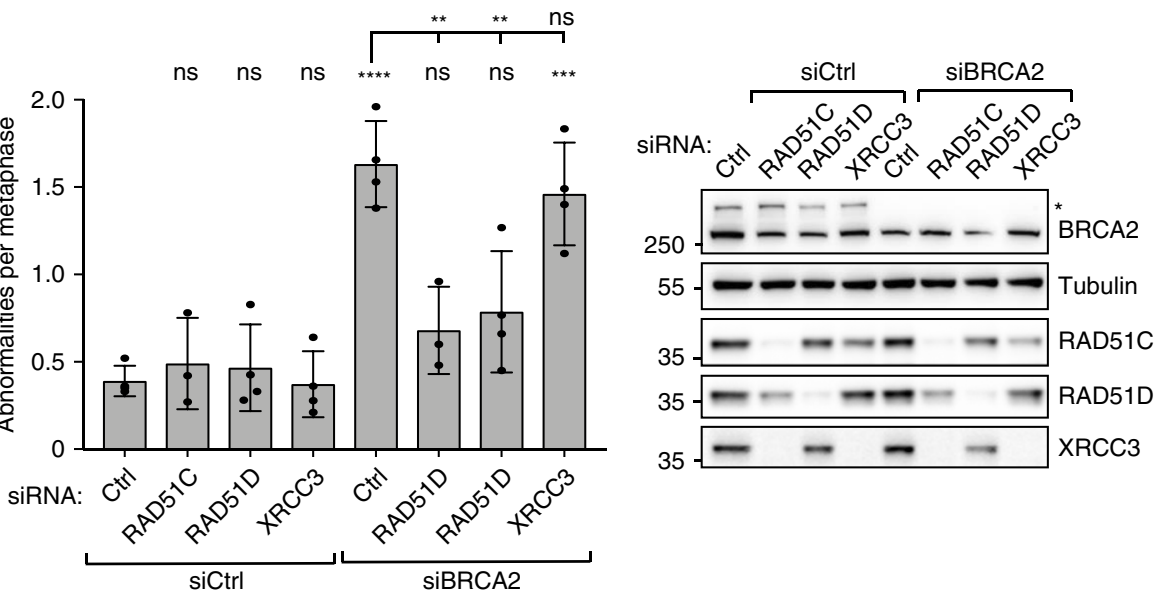

b
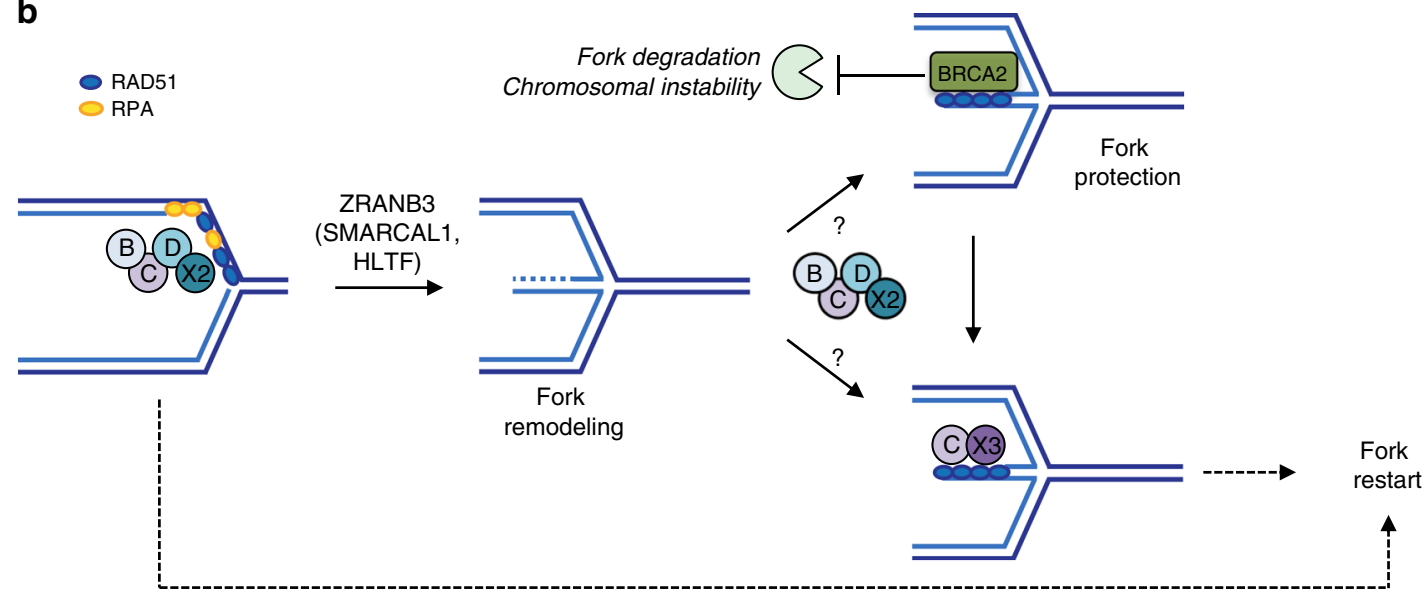

Fast restart (repriming?)

Fig. 5 BCDX2, but not CX3, promotes chromosomal instability in BRCA2-defective cells. a Metaphase spread analysis for detecting chromosomal aberrations in U2OS cells double transfected with a control siRNA (Ctrl) or with siRNAs targeting RAD51C, RAD51D, and XRCC3 for 60 h and with BRCA2 siRNA for $48 \mathrm{~h}$ and treated with $4 \mathrm{mM} \mathrm{HU}$ for $5 \mathrm{hr}$. Left: representative metaphase spread image (scale-bar, $5 \mu \mathrm{m}$ ); $1,2,3$ representative breaks and 4 representative intact chromosome. Center: number of chromosomal abnormalities for each genetic condition. The graph-bar depicts mean and SD from at least three independent experiments. Statistical analysis: one-way ANOVA followed by Bonferroni test; ns not significant; ${ }^{\star \star} p$-value $=0.0012$ ( 5 versus 6 ) and 0.0020 (5 versus 7); ${ }^{\star \star \star} p$-value $=0.0001 ;{ }^{\star \star \star \star} p$-value $<0.0001$. Right: levels of indicated proteins, assessed by western blot; Tubulin, loading control; asterisk, specific band. Multiple gels/blots were processed in parallel, ensuring equal and comparable loading across gels. Source data are provided in the Source Data file. b Proposed model for the sequential role of BCDX2 and CX3 paralog complexes in replication fork remodeling, protection and restart: the BCDX2 complex helps RAD51 and ZRANB3 in driving fork remodeling. During fork stalling, regressed arms are protected from unscheduled nucleolytic degradation by BRCA2-mediated stabilization of RAD51 nucleofilament. Preventing fork remodeling by BCDX2 inactivation suppresses genetic instability of BRCA2-deficient cells upon sustained replicative stress. Similarly to RAD51, BCDX2 may be involved in fork protection (and possibly fork restart), but this role may be genetically masked by its upstream role in promoting fork reversal. Once DNA synthesis can resume, the CX3 complex promotes reversed fork restart, likely by engaging RAD51-bound regressed arms in efficient strand invasion events. In absence of fork remodeling, stalled forks undergo a fast, but possibly inaccurate restart mode, likely driven by repriming events.

Although both BRCA2 and XRCC3 were shown to play a role downstream of fork remodeling, they display rather different, yet possibly coordinated reversal-dependent functions. While BRCA2 defects lead to reversed fork degradation, but mild fork restart defects $13,17,20$, XRCC3 inactivation does not impair fork stability, but severely impairs single-fork reactivation once the stalling agent has been removed (Fig. 4), thus respectively handling a static (fork protection) and dynamic (fork restart) replication stress response. While the role of BRCA2 in fork protection is clearly linked to RAD51 nucleofilament stabilization on the regressed $\operatorname{arm}^{14,17}$, we propose that XRCC3 assists fork restart by promoting RAD51mediated strand invasion of parental DNA by the regressed arm, which may be important for at least a subset of fork restart events. Consistently, the human CX3 complex is specifically observed to promote RAD51 nucleofilament remodeling and stability, thereby promoting strand invasion (Lumir Krejci, personal communication), similarly to what was previously reported for the unique RAD51 paralogs complex in C. elegans ${ }^{41}$. Alternatively, CX3 may facilitate the recruitment of specialized factors, catalyzing fork restoration via branch migration or controlled fork processing. Overall, several different scenarios could be envisioned to explain CX3 contribution to fork restart, and only a complete biochemical reconstitution of this reaction in the future-including known fork restart activities-will possibly clarify.

Importantly, BCDX2 inactivation, but not XRCC3 downregulation, rescued chromosomal abnormalities upon fork stalling in BRCA2-defective cells. These data are aligned with previous findings with DNA translocase inactivation ${ }^{22}$ and reinforce the concept of replication fork reversal as a double-edged sword in genome maintenance, requiring tight biological control. While on 
one hand fork reversal represents an important mechanism to avoid replication stress-associated chromosomal breakage $e^{5,8,58}$, it can also drive clinically relevant pathogenic transactions in the absence of functional fork protection and restart mechanisms ${ }^{10,13,20,22}$. Such data suggest that multiple defects in the same remodeling pathway may occasionally combine, leading to different outcomes in terms of genome stability. As reversaldependent degradation was shown to be a major molecular determinant of chemosensitivity and -resistance in HR-defective tumors $^{20}$, it will be essential to test the molecular phenotypes upon cancer-associated mutations in RAD51 paralogs, and thereby to possibly establish predictive parameters for chemosensitivity in this subset of HR-defective tumors.

\section{Methods}

Cell culture and cell lines. Human U2OS and hTERT-RPE-1 cells were grown in Dulbecco's modified Eagle's medium (DMEM) supplemented with $10 \%$ fetal bovine serum (FBS; GIBCO/Thermo Fisher Scientific) and 1\% penicillinstreptomycin antibiotics ( $100 \mathrm{U} / \mathrm{mL}$ penicillin and $100 \mu \mathrm{g} / \mathrm{mL}$ streptomycin) under standard cell culture conditions (humidified atmosphere, $6 \% \mathrm{CO}_{2}$ ) at $37^{\circ} \mathrm{C}$.

ZRANB3-proficient and Knock-Out U2OS cells were kindly provided by Dr. David Cortez.

RAD51 paralog CRISPSR-Cas9-based Knock-Out U2OS cells have been generated and genetically characterized as recently reported ${ }^{46}$.

siRNA transfection and sequences. The QIBC-based siRNA screen was performed by reverse transfection of U2OS cells in CELLSTAR 96-well plates (Greiner Bio-One) for $48 \mathrm{~h}$ at a cell density of 5000 cells per well at the time of transfection with Ambion Silencer Select siRNAs at a final concentration of $5 \mathrm{nM}$ using HiPerFect reagent (Qiagen).

Individual siRNA transfections were performed using Lipofectamine RNAiMAX (ThermoFisher Scientific) at a concentration of $10 \mathrm{nM}$ (Ambion Silencer Select) or $40 \mathrm{nM}$ (Microsynth AG) according to manufacturer's instruction and experiments performed between 48 and $72 \mathrm{~h}$ post transfection as indicated.

The oligonucleotides used for individual assays in this study are presented in Supplementary Table 2. Unless stated otherwise in the Figure legend siCtrl (as negative siRNA control), siRAD51C \#2, siRAD51D \#1, and XRCC3 \#1 have been used.

Drugs and reagents. The following reagents were used to treat the cells for the indicated time at the indicated final concentrations before collection: Hydroxyurea (HU; H8627, Sigma-Aldrich) was prepared in double-distilled H2O to obtain a $1 \mathrm{M}$ stock and stored at $-20^{\circ} \mathrm{C}$; Mirin (M9948, Sigma-Aldrich) was dissolved in DMSO to yield a $50 \mathrm{mM}$ stock and aliquots were stored at $-80^{\circ} \mathrm{C}$; Camptothecin (CPT; C9911, Sigma-Aldrich) was dissolved in DMSO to yield a $5 \mathrm{mM}$ stock (freshly made); Nocodazole (M1404, Sigma-Aldrich) was prepared in DMSO at the final concentration of $1 \mathrm{mg} \mathrm{ml}^{-1}$, and aliquots were stored at $-20^{\circ} \mathrm{C}$.

Immunoblotting. Cell were washed in cold PBS and lysed in NP-40 buffer (50 mM Tris- $\mathrm{HCl}$ [pH 7.5], $150 \mathrm{mM} \mathrm{NaCl}, 1 \% \mathrm{NP}-40)$ supplemented with $1 \times$ protease inhibitor cocktail (cOmplete, Roche) and phosphatase inhibitors (20 mM NaF, $1 \mathrm{mM} \mathrm{Na}_{3} \mathrm{VO}_{4}$ and $5 \mathrm{mM} \mathrm{Na}_{4} \mathrm{P}_{2} \mathrm{O}_{7}$ ). Cell extract were diluted with NuPAGE LDS sample buffer (Thermo Fisher Scientific) containing DTT and heated at $60^{\circ} \mathrm{C}$ for 10 min. Proteins, together with the PageRuler Plus Prestained Protein Ladder (Thermo Fisher Scientific, 26620), were resolved on NuPAGE 4-12\%, Bis-Tris or 3-8\% Tris-Acetate gels (Thermo Fisher Scientific) using MOPS and Tris-Acetate SDS running buffers respectively, and transferred on nitrocellulose membranes. Membranes were blocked with TBS containing Tween-20 (0.1\%) and 5\% milk powder for $1 \mathrm{~h}$ at room temperature (RT), incubated with the indicated primary antibodies overnight at $4{ }^{\circ} \mathrm{C}$ and secondary antibodies for $1 \mathrm{~h}$ at RT. Protein were visualized using WesternBright ECL reagent (Advansta) and the Fusion Solo S imaging system (Vilber Lourmat). Uncropped and unprocessed scans of each blot are provided in the Source Data file.

The following primary antibodies were used: RAD51B mouse (sc-377192, Santa Cruz Biotechnology, 1:200), RAD51C rabbit (ab95069, Abcam, 1:2000), RAD51D rabbit (ab202063, Abcam, 1:500), XRCC2 mouse (sc-365854, Santa Cruz Biotechnology, 1:200), XRCC3 mouse (sc-271714, Santa Cruz Biotechnology, 1:200), BRCA2 mouse (OP-95, EMD Millipore, 1:500), RAD51 rabbit (Bioacademia 70-002, 1:5000), ZRANB3 rabbit (23111-1-AP, Proteintech, 1:500), KU70 mouse (ab202022, Abcam, 1:2000), and a-Tubulin mouse (T9026, Sigma-Aldrich, 1:10000).

Immunostaining. Cells were grown on glass coverslips or 96-well plates, washed in PBS, pre-extracted for $5 \mathrm{~min}$ at $4{ }^{\circ} \mathrm{C}$ in CSK buffer (10 mM Hepes-KOH [pH 7.4], $300 \mathrm{mM}$ sucrose, $50 \mathrm{mM} \mathrm{NaCl}, 3 \mathrm{mM} \mathrm{MgCl}_{2}, 1 \mathrm{mM}$ EGTA, $0.5 \%$ Triton X-100), fixed in $4 \%$ paraformaldehyde in PBS for $12 \mathrm{~min}$ at RT, washed twice in PBS, permeabilized in PBS supplemented with $0.3 \%$ Triton X-100, washed twice in PBS and blocked with $3 \%$ BSA in PBS twice for 15 min. Rabbit polyclonal RAD51 antibody (Bioacademia 70-002, 1:2000) and secondary antibody (Alexa fluorophores, Life Technologies) were diluted in $1.5 \%$ BSA in PBS and incubation were, respectively, performed for 2 or $1 \mathrm{~h}$ at RT. Coverslips were washed with PBS supplemented with $0.05 \%$ Tween-20 and incubated for 10 min with PBS containing 4',6-Diamidino-2-Phenylindole Dihydrochloride (DAPI, $0.5 \mu \mathrm{g} / \mathrm{mL}$ ) at RT to stain DNA. Following three washing steps in PBS, coverslips were rinsed with distilled water, air-dried and mounted with ProLong Gold AntiFade (Thermo Fisher Scientific). When indicated the Click-iT EdU Alexa Fluor Imaging Kit (Thermo Fisher Scientific) was used for EdU detection according to manufacturer's instruction.

Quantitative image-based cytometry (QIBC). QIBC experiments were performed on an Olympus ScanR Screening System equipped with an inverted motorized Olympus IX83 microscope, a motorized stage, IR-laser hardware autofocus, a fast emission filter wheel with single band emission filters, and a digital monochrome Hamamatsu ORCA-FLASH 4.0 V2 sCMOS camera $(2048 \times 2048$ pixel, pixel size $6.5 \times 6.5 \mu \mathrm{m}, 12$ bit dynamics), as described previously ${ }^{70,71}$. For each condition, image information of large cohorts of cells (typically at least 500 cells for the UPLSAPO $\times 40$ objective (NA 0.9) and at least 2000 cells for the UPLSAPO $\times 20$ objective (NA 0.75 ) ) was acquired under non-saturating conditions. Identical settings were applied to all samples within one experiment. Images were analyzed with the Olympus ScanR Image Analysis Software, a dynamic background correction was applied, nuclei segmentation was performed using an integrated intensity-based object detection module using the DAPI signal, and foci segmentation was performed using an integrated spot-detection module. All downstream analyses were focused on properly detected nuclei containing a $2 \mathrm{C}-4 \mathrm{C}$ DNA content as measured by total and mean DAPI intensities. Fluorescence intensities were quantified and are depicted as arbitrary units. Color-coded scatterplots of asynchronous cell populations were generated with Spotfire data visualization software (TIBCO). Within one experiment, similar cell numbers were compared for the different conditions. For visualizing discrete data in scatterplots, mild jittering (random displacement of data points along the discrete data axes) was applied in order to demerge overlapping data points. For the siRNA screen, genes were ranked according to a $\mathrm{z}$-score $(\mathrm{z}=(\mathrm{x}-\mu) / \sigma$ with $\mathrm{x}$ being the mean number of RAD51 foci per cell for each knockdown, $\mu$ being the mean number of RAD51 foci per cell across all conditions, and $\sigma$ being the standard deviation of the mean number of RAD51 foci per cell across all conditions). Representative scatterplots and quantifications of independent experiments are shown. Representative images, in which the individual color channels have been adjusted for brightness and contrast, accompany selected quantifications.

DNA fiber spreading analysis. Asynchronously growing cells were labeled with the thymidine analogues 5-Chloro- $2^{\prime}$-deoxyuridine (CldU, $\left.30 \mu \mathrm{M}\right)$, washed 3 times with PBS, followed by 5 -Iodo-2'-deoxyuridine (IdU, $250 \mu \mathrm{M})$ and treated with $\mathrm{HU}$ and CPT as indicated ${ }^{58}$. The cells were quickly trypsinized and resuspended in ice-cold PBS at $2.5 \times 10^{5}$ cells per ml. The labeled cells were diluted 1:1 with unlabeled cells and $3 \mu \mathrm{l}$ of cells were mixed with $7.5 \mu \mathrm{l}$ of lysis buffer $(200 \mathrm{mM}$ Tris- $\mathrm{HCl}$ [pH 7.5], $50 \mathrm{mM}$ EDTA, $0.5 \%(\mathrm{w} / \mathrm{v}) \mathrm{SDS}$ ) on a glass slide. After $9 \mathrm{~min}$, the slides were tilted at $15^{\circ}-45^{\circ}$, and the resulting DNA spreads were air-dried, fixed in 3:1 methanol/acetic acid overnight at $4{ }^{\circ} \mathrm{C}$. The DNA fibers were denatured with $2.5 \mathrm{M} \mathrm{HCl}$ for $1 \mathrm{~h}$, washed with PBS and blocked with $2 \%$ BSA in PBS supplemented with $0.1 \%$ Tween20 for $40 \mathrm{~min}$. The newly replicated CldU and IdU tracks were labeled (for $2.5 \mathrm{~h}$ in the dark, at RT) with anti-BrdU/CldU antibodies recognizing CldU (ab6326, Abcam, rat, 1:500) and BrdU/IdU (347580, Becton Dickinson, mouse, 1:100), respectively. After washing $5 \times 3 \mathrm{~min}$ in PBS supplemented with $0.2 \%$ Tween-20, the following secondary antibodies were used (incubated for $2 \mathrm{~h}$ in the dark, at RT): anti-mouse Alexa 488 (Molecular Probes, 1:300), anti-rat Cy3 (Jackson Immunoresearch, 1:150). After washing $5 \times 3$ min each in PBS supplemented with $0.2 \%$ Tween-20 the slides were airdried completely and mounted with $20 \mathrm{uL} /$ slide ProLong Gold AntiFade (Thermo Fisher Scientific). Images were acquired using an Olympus IX81 fluorescence microscope equipped with a CCD camera (Orca AG, Hamamatsu). CldU and IdU tract lengths were measured using the line tool in ImageJ64 software. In all the fork restart experiments (Fig. 4), defective fork restart (i.e., fork stalling) was defined as a ratio (length of green vs red) $<0.1$.

Electron microscopic analysis of genomic DNA. Following the depletion of the protein of interest, asynchronous subconfluent cells were treated with $50 \mathrm{nM} \mathrm{CPT}$ for $1 \mathrm{~h}$ or $4 \mathrm{mM} \mathrm{HU}$ for $5 \mathrm{~h}$. Where indicated, cells were pretreated with $50 \mu \mathrm{M}$ Mirin for $1 \mathrm{~h}$. Cells were collected, resuspended in ice-cold PBS and crosslinked with 4,5', 8trimethylpsoralen $(10 \mu \mathrm{g} / \mathrm{ml}$ final concentration), followed by irradiation pulses with UV $365 \mathrm{~nm}$ monochromatic light (UV Stratalinker 1800; Agilent Technologies). For DNA extraction ${ }^{66}$, cells were lysed (1.28 M sucrose, $40 \mathrm{mM}$ Tris- $\mathrm{HCl}$ [pH 7.5], $20 \mathrm{mM} \mathrm{MgCl}_{2}$, and 4\% Triton X-100; Qiagen) and digested ( $800 \mathrm{mM}$ guanidine- $\mathrm{HCl}$, $30 \mathrm{mM}$ Tris-HCl [pH 8.0], $30 \mathrm{mM}$ EDTA [pH 8.0], 5\% Tween-20, and 0.5\% Triton $\mathrm{X}-100)$ at $50^{\circ} \mathrm{C}$ for $2 \mathrm{~h}$ in presence of $1 \mathrm{mg} / \mathrm{ml}$ proteinase $\mathrm{K}$. The DNA was purified using chloroform/isoamylalcohol (24:1) and precipitated in 0.7 volume of isopropanol. Finally, the DNA was washed with $70 \% \mathrm{EtOH}$ and resuspended in $200 \mu \mathrm{l}$ TE (Tris-EDTA) buffer. $100 \mathrm{U}$ of restriction enzyme (PvuII high fidelity, New 
England Biolabs) were used to digest $6 \mu \mathrm{g}$ of mammalian genomic DNA for $5 \mathrm{~h}$. RNase A (Sigma-Aldrich, R5503) to a final concentration of $250 \mathrm{ug} / \mathrm{ml}$ was added for the last $2 \mathrm{~h}$ of this incubation. The digested DNA was transferred into Microcon DNA Fast Flow centrifugal filters (Merck MRCF0R100) and washed two times with $200 \mathrm{ul}$ TE. Ultimately, the digested DNA was concentrated and recovered by inverting the filters. The Benzyldimethylalkylammonium chloride (BAC) method was used to spread the DNA on the water surface and then load it on carbon-coated 400-mesh nickel grids (G2400N, Plano Gmbh). Subsequently, DNA was coated with platinum using a High Vacuum Evaporator BAF060 (Leica). The grids were scanned using a transmission electron microscope (Tecnai G2 Spirit; FEI; LaB6 filament; high tension $\leq 120 \mathrm{kV}$ ) and pictures were acquired with a side mount charge-coupled device camera $(2600 \times 4000$ pixels; Orius 1000 ; Gatan, Inc.) and processed with DigitalMicrograph Version 1.83.842 (Gatan, Inc.). For each experimental condition at least70 replication fork molecules were analyzed in three different biological replicates by using ImageJ64

\section{Chromosomal breakage and abnormalities by metaphase spreading. After} transfection with the indicated siRNAs, cells were treated for $5 \mathrm{~h}$ with $4 \mathrm{mM}$ HU. The compound was washed off three times with $1 \times$ PBS, upon which cells were released into fresh medium containing $200 \mathrm{ng} / \mathrm{ml}$ nocodazole for $16 \mathrm{~h}$. Cells were harvested and swollen with $75 \mathrm{mM} \mathrm{KCl}$ for $20 \mathrm{~min}$ at $37^{\circ} \mathrm{C}$. Swollen mitotic cells were collected and fixed with methanol:acetic acid (3:1). The fixing step was repeated two times. Fixed cells were dropped onto prehydrated glass slides and air-dried overnight. The following day, slides were mounted with Vectashield medium containing DAPI. Microscopy was performed on a Leica DM6 B upright digital research microscope equipped with a DFC360 FX Leica camera. Images were analyzed using ImageJ64 and visible chromatid breaks/gaps were counted. For each experimental condition at least 50 metaphases were analyzed in three different biological replicates.

Statistical analysis. For QIBC analysis, between 9 and 15 images per condition, depending on the microscope objective used and the cell confluence were acquired in an unbiased fashion from asynchronous cell populations grown on glass coverslips or multi-well plates. Typically, between 2000 and 5000 cells per condition were analyzed and representative single-cell data of cell cohorts of comparable size are shown as two-dimensional cell-cycle-resolved or one-dimensional scatterplots.

For DNA fiber length measurements, at least 150 fibers were scored for each condition and every experiment was repeated at least twice. The results are depicted as median plus 10-90 percentile whisker plots and Kurskal-Wallis test was used for statistical analysis.

In all the other experiments, including DNA fiber analysis of fork restart, the statistical significance for three different biological replicates was determined by one-way ANOVA followed by Bonferroni test.

GraphPad Prism7 for MacOSX was used for all statistical analyses.

Reporting summary. Further information on research design is available in the Nature Research Reporting Summary linked to this article.

\section{Data availability}

Source data are provided with this paper. All other data that support the findings of this study are available from the authors upon reasonable request.

Received: 14 October 2019; Accepted: 16 June 2020;

Published online: 15 July 2020

\section{References}

1. Berti, M. \& Vindigni, A. Replication stress: getting back on track. Nat. Struct. Mol. Biol. 23, 103-109 (2016).

2. Forment, J. V. \& O'Connor, M. J. Targeting the replication stress response in cancer. Pharmacol. Ther. 188, 155-167 (2018).

3. Follonier, C., Oehler, J., Herrador, R. \& Lopes, M. Friedreich's ataxia-associated GAA repeats induce replication-fork reversal and unusual molecular junctions. Nat. Struct. Mol. Biol. 20, 486-494 (2013).

4. Neelsen, K. J., Zanini, I. M. Y., Herrador, R. \& Lopes, M. Oncogenes induce genotoxic stress by mitotic processing of unusual replication intermediates. $J$. Cell Biol. 200, 699-708 (2013).

5. Zellweger, R. et al. Rad51-mediated replication fork reversal is a global response to genotoxic treatments in human cells. J. Cell Biol. 208, 563-579 (2015).

6. Berti, M. et al. Human RECQ1 promotes restart of replication forks reversed by DNA topoisomerase I inhibition. Nat. Struct. Mol. Biol. 20, 347-354 (2013).

7. Thangavel, S. et al. DNA2 drives processing and restart of reversed replication forks in human cells. J. Cell Biol. 208, 545-562 (2015).

8. Neelsen, K. J. \& Lopes, M. Replication fork reversal in eukaryotes: from dead end to dynamic response. Nat. Rev. Mol. Cell Biol. 16, 207-220 (2015).
9. Cortez, D. Replication-coupled DNA repair. Mol. Cell 74, 866-876 (2019).

10. Berti, M., Cortez, D. \& Lopes, M. The plasticity of DNA replication forks in response to clinically relevant genotoxic stress. Nat. Rev. Mol. Cell Biol. 10.1038-s41580-020-0257-5 (2020).

11. Betous, R. et al. SMARCAL1 catalyzes fork regression and Holliday junction migration to maintain genome stability during DNA replication. Genes Dev. 26, 151-162 (2012).

12. Kile, A. C. et al. HLTF's ancient HIRAN domain binds $3^{\prime}$ DNA ends to drive replication fork reversal. Mol. Cell 58, 1090-1100 (2015).

13. Lemacon, D. et al. MRE11 and EXO1 nucleases degrade reversed forks and elicit MUS81-dependent fork rescue in BRCA2-deficient cells. Nat. Commun. 8, 860 (2017).

14. Mijic, S. et al. Replication fork reversal triggers fork degradation in BRCA2defective cells. Nat. Commun. 8, 859 (2017).

15. Vujanovic, M. et al. Replication fork slowing and reversal upon genotoxic stress require PCNA polyubiquitination and ZRANB3 DNA translocase activity. Mol. Cell 67, 882-890 (2017).

16. Bhat, K. P. \& Cortez, D. RPA and RAD51: fork reversal, fork protection, and genome stability. Nat. Struct. Mol. Biol. 25, 446-453 (2018).

17. Schlacher, K. et al. Double-strand break repair-independent role for BRCA2 in blocking stalled replication fork degradation by MRE11. Cell 145, 529-542 (2011).

18. Schlacher, K., Wu, H. \& Jasin, M. A distinct replication fork protection pathway connects Fanconi anemia tumor suppressors to RAD51-BRCA1/2. Cancer Cell 22, 106-116 (2012).

19. Wang, A. T. et al. A dominant mutation in human RAD51 reveals its function in DNA interstrand crosslink repair independent of homologous recombination. Mol. Cell 59, 478-490 (2015).

20. Chaudhuri, A. R. et al. Replication fork stability confers chemoresistance in BRCA-deficient cells. Nature 535, 382-387 (2016).

21. Przetocka, S. et al. CtIP-mediated fork protection synergizes with BRCA1 to suppress genomic instability upon DNA replication stress. Mol. Cell 72, 568-582.e6 (2018)

22. Taglialatela, A. et al. Restoration of replication fork stability in BRCA1- and BRCA2-deficient cells by inactivation of SNF2-family fork remodelers. Mol. Cell 68, 414-430.e8 (2017).

23. Kolinjivadi, A. M. et al. Smarcal1-mediated fork reversal triggers Mre11dependent degradation of nascent DNA in the absence of Brca2 and stable Rad51 nucleofilaments. Mol. Cell 67, 867-881.e7 (2017).

24. Malacaria, E. et al. Rad52 prevents excessive replication fork reversal and protects from nascent strand degradation. Nat. Commun. 10, 1412-1419 (2019)

25. Sullivan, M. R. \& Bernstein, K. A. RAD-ical new insights into RAD51 regulation. Genes (Basel) 9, 629 (2018).

26. Liu, T., Wan, L., Wu, Y., Chen, J. \& Huang, J. hSWS1.SWSAP1 is an evolutionarily conserved complex required for efficient homologous recombination repair. J. Biol. Chem. 286, 41758-41766 (2011).

27. Abreu, C. M. et al. Shu complex SWS1-SWSAP1 promotes early steps in mouse meiotic recombination. Nat. Commun. 9, 3961 (2018).

28. Yonetani, Y. et al. Differential and collaborative actions of Rad51 paralog proteins in cellular response to DNA damage. Nucleic Acids Res. 33, 4544-4552 (2005).

29. Yokoyama, H. et al. Preferential binding to branched DNA strands and strand-annealing activity of the human Rad51B, Rad51C, Rad51D and Xrcc2 protein complex. Nucleic Acids Res. 32, 2556-2565 (2004).

30. Liu, N., Schild, D., Thelen, M. P. \& Thompson, L. H. Involvement of Rad51C in two distinct protein complexes of Rad51 paralogs in human cells. Nucleic Acids Res. 30, 1009-1015 (2002).

31. Masson, J. Y. et al. Identification and purification of two distinct complexes containing the five RAD51 paralogs. Genes Dev. 15, 3296-3307 (2001)

32. Miller, K. A. et al. RAD51C interacts with RAD51B and is central to a larger protein complex in vivo exclusive of RAD51. J. Biol. Chem. 277, 8406-8411 (2002).

33. Takata, M. et al. Chromosome instability and defective recombinational repair in knockout mutants of the five Rad51 paralogs. Mol. Cell Biol. 21, 2858-2866 (2001).

34. Godthelp, B. C. et al. Mammalian Rad51C contributes to DNA cross-link resistance, sister chromatid cohesion and genomic stability. Nucleic Acids Res. 30, 2172-2182 (2002).

35. Bishop, D. K. et al. Xrcc3 is required for assembly of Rad51 complexes in vivo. J. Biol. Chem. 273, 21482-21488 (1998).

36. Liu, N. et al. XRCC2 and XRCC3, new human Rad51-family members, promote chromosome stability and protect against DNA cross-links and other damages. Mol. Cell 1, 783-793 (1998).

37. Pierce, A. J., Johnson, R. D., Thompson, L. H. \& Jasin, M. XRCC3 promotes homology-directed repair of DNA damage in mammalian cells. Genes Dev. 13 2633-2638 (1999)

38. French, C. A. et al. Role of mammalian RAD51L2 (RAD51C) in recombination and genetic stability. J. Biol. Chem. 277, 19322-19330 (2002) 
39. Johnson, R. D., Liu, N. \& Jasin, M. Mammalian XRCC2 promotes the repair of DNA double-strand breaks by homologous recombination. Nature 401, 397-399 (1999).

40. Liu, J. et al. Rad51 paralogues Rad55-Rad57 balance the antirecombinase Srs2 in Rad51 filament formation. Nature 479, 245-248 (2011).

41. Taylor, M. R. G. et al. Rad51 paralogs remodel pre-synaptic Rad51 filaments to stimulate homologous recombination. Cell 162, 271-286 (2015).

42. Taylor, M. R. G. et al. A polar and nucleotide-dependent mechanism of action for RAD51 paralogs in RAD51 filament remodeling. Mol. Cell 64, 926-939 (2016).

43. Jensen, R. B., Ozes, A., Kim, T., Estep, A. \& Kowalczykowski, S. C. BRCA2 is epistatic to the RAD51 paralogs in response to DNA damage. DNA Repair (Amst.) 12, 306-311 (2013).

44. Roy, R., Chun, J. \& Powell, S. N. BRCA1 and BRCA2: different roles in a common pathway of genome protection. Nat. Rev. Cancer 12, 68-78 (2012).

45. Chun, J., Buechelmaier, E. S. \& Powell, S. N. Rad51 paralog complexes BCDX2 and CX3 act at different stages in the BRCA1-BRCA2-dependent homologous recombination pathway. Mol. Cell Biol. 33, 387-395 (2013).

46. Garcin, E. B. et al. Differential requirements for the RAD51 paralogs in genome repair and maintenance in human cells. PLoS Genet. 15:e1008355 https://doi.org/10.1371/journal.pgen.1008355 (2019).

47. Akbari, M. R. et al. RAD51C germline mutations in breast and ovarian cancer patients. Breast Cancer Res. 12, 404 (2010).

48. Loveday, C. et al. Germline mutations in RAD51D confer susceptibility to ovarian cancer. Nat. Genet. 43, 879-882 (2011).

49. Loveday, C. et al. Germline RAD51C mutations confer susceptibility to ovarian cancer. Nat. Genet. 44, 475-476 (2012).

50. Orr, N. et al. Genome-wide association study identifies a common variant in RAD51B associated with male breast cancer risk. Nat. Genet. 44, 1182-1184 (2012).

51. Park, D. J. et al. Rare mutations in XRCC2 increase the risk of breast cancer. Am. J. Hum. Genet. 90, 734-739 (2012).

52. Park, J.-Y. et al. Complementation of hypersensitivity to DNA interstrand crosslinking agents demonstrates that XRCC2 is a Fanconi anaemia gene. J. Med. Genet. 53, 672-680 (2016).

53. Vaz, F. et al. Mutation of the RAD51C gene in a Fanconi anemia-like disorder. Nat. Genet. 42, 406-409 (2010).

54. Somyajit, K., Saxena, S., Babu, S., Mishra, A. \& Nagaraju, G. Mammalian RAD51 paralogs protect nascent DNA at stalled forks and mediate replication restart. Nucleic Acids Res. 43, 9835-9855 (2015).

55. Henry-Mowatt, J. et al. XRCC 3 and Rad51 modulate replication fork progression on damaged vertebrate chromosomes. Mol. Cell 11, 1109-1117 (2003).

56. Sugimura, K., Takebayashi, S., Taguchi, H., Takeda, S. \& Okumura, K. PARP1 ensures regulation of replication fork progression by homologous recombination on damaged DNA. J. Cell Biol. 183, 1203-1212 (2008).

57. Saxena, S., Somyajit, K. \& Nagaraju, G. XRCC2 regulates replication fork progression during dNTP alterations. Cell Rep. 25, 3273-3282.e6 (2018).

58. Ray Chaudhuri, A. et al. Topoisomerase I poisoning results in PARP-mediated replication fork reversal. Nat. Struct. Mol. Biol. 19, 417-423 (2012).

59. Lossaint, G. et al. FANCD2 binds MCM proteins and controls replisome function upon activation of s phase checkpoint signaling. Mol. Cell 51, 678-690 (2013).

60. Dungrawala, H. et al. The replication checkpoint prevents two types of fork collapse without regulating replisome stability. Mol. Cell 59, 998-1010 (2015).

61. Alabert, C. et al. Nascent chromatin capture proteomics determines chromatin dynamics during DNA replication and identifies unknown fork components. Nat. Cell Biol. 16, 281-293 (2014).

62. Altmeyer, M. et al. The chromatin scaffold protein SAFB1 renders chromatin permissive for DNA damage signaling. Mol. Cell 52, 206-220 (2013).

63. Toledo, L. I. et al. ATR prohibits replication catastrophe by preventing global exhaustion of RPA. Cell 155, 1088-1103 (2013).

64. Martino, J. et al. The human Shu complex functions with PDS5B and SPIDR to promote homologous recombination. Nucleic Acids Res. 47, 10151-10165 (2019).

65. Vujanovic, M. et al. Replication fork slowing and reversal upon DNA damage require PCNA polyubiquitination and ZRANB3 DNA translocase activity. Mol. Cell 67, 882-890.e5 (2017).

66. Zellweger, R. \& Lopes, M. Dynamic architecture of eukaryotic DNA replication forks in vivo, visualized by electron microscopy. Methods Mol. Biol. 1672, 261-294 (2018).

67. Bugreev, D. V., Rossi, M. J. \& Mazin, A. V. Cooperation of RAD51 and RAD54 in regression of a model replication fork. Nucleic Acids Res. 39, 2153-2164 (2011).
68. Betous, R. et al. Substrate-selective repair and restart of replication forks by DNA translocases. Cell Rep. 3, 1958-1969 (2013).

69. Liu, Y., Masson, J.-Y., Shah, R., O’Regan, P. \& West, S. C. RAD51C is required for Holliday junction processing in mammalian cells. Science 303, 243-246 (2004).

70. Teloni, F. et al. Efficient Pre-mRNA Cleavage Prevents Replication-StressAssociated Genome Instability. Mol. Cell 73, 670-683.e12 (2019).

71. Michelena, J. et al. Analysis of PARP inhibitor toxicity by multidimensional fluorescence microscopy reveals mechanisms of sensitivity and resistance. Nat. Commun. 9, (2018).

\section{Acknowledgements}

We thank the Center for Microscopy and Image Analysis of the University of Zurich for assistance with microscopy and imaging analysis. We are grateful to Eli Rothenberg, Huijun Xue, Petr Cejka, Swagata Halder and members of the Lopes and Altmeyer labs for technical assistance and fruitful discussions, and to Jean-Yves Masson for critical reading of the manuscript. Work in the Lopes lab was supported by the SNF grant 31003A_169959, the ERC Consolidator Grant 617102 and the Swiss Cancer League grant KFS-3967-08-2016 to M.L. M.B. was also supported by the H2020 Marie Sklodowska-Curie postdoctoral fellowship 704817. Work in the Altmeyer lab was supported by SNF grants PP00P3_150690 and PP00P3_179057 and the European Research Council (ERC) under the European Union's Horizon 2020 research and innovation program ERC Starting Grant 714326 to M.A. F.T. was also supported by a Candoc fellowship from UZH. S.G and M.M. were supported by CRCM state subsidies from CNRS, Inserm, Institute Paoli-Calmettes and AixMarseille University, and the French National League against Cancer. E.B.G. was supported by Postdoctoral fellowships from the French National League against Cancer and from AixMarseille University Foundation.

\section{Author contributions}

M.B. and F.T. designed and performed the QIBC-based screen and conducted validation experiments; M.B., S.M., and J.K. performed most DNA fiber assays, while S.U. and S.M. performed most EM experiments, assisted by M.B., J.F., J.K., J.A.S., and M.D.P.; J.K. and J.A.S. performed the metaphase spread analysis. E.B.G., S.G., and M.M. produced all CRISPR-KO cell lines and shared them ahead of publication. M.L. designed the project and wrote the first manuscript draft, helped by M.B., F.T., and M.A.; M.L. and M.A. supervised the project; all authors read and provided feedback on the manuscript.

\section{Competing interests}

The authors declare no competing interests.

\section{Additional information}

Supplementary information is available for this paper at https://doi.org/10.1038/s41467 020-17324-z.

Correspondence and requests for materials should be addressed to M.A. or M.L.

Peer review information Nature Communications thanks Amir Aharoni, Claudia Wiese and the other anonymous reviewer(s) for their contribution to the peer review of this work. Peer reviewer reports are available.

Reprints and permission information is available at http://www.nature.com/reprints

Publisher's note Springer Nature remains neutral with regard to jurisdictional claims in published maps and institutional affiliations.

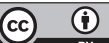

Open Access This article is licensed under a Creative Commons Attribution 4.0 International License, which permits use, sharing, adaptation, distribution and reproduction in any medium or format, as long as you give appropriate credit to the original author(s) and the source, provide a link to the Creative Commons license, and indicate if changes were made. The images or other third party material in this article are included in the article's Creative Commons license, unless indicated otherwise in a credit line to the material. If material is not included in the article's Creative Commons license and your intended use is not permitted by statutory regulation or exceeds the permitted use, you will need to obtain permission directly from the copyright holder. To view a copy of this license, visit http://creativecommons.org/ licenses/by/4.0/.

(C) The Author(s) 2020 